\title{
Goat milk during iron repletion improves bone turnover impaired by severe iron deficiency
}

\author{
J. Díaz-Castro,, ${ }^{\star 1}$ M. Ramírez López-Frías, ${ }^{*}$ M. S. Campos, ${ }^{\star}$ M. López-Frías, ${ }^{\star}$ M. J. M. Alférez, ${ }^{\star}$ T. Nestares, ${ }^{*}$ \\ E. Ortega,† and I. López-Aliaga* \\ *Department of Physiology and Institute of Nutrition and Food Technology, University of Granada, E-18071 Granada, Spain \\ †Department of Biochemistry and Biology Molecular, University of Granada, E-18071 Granada, Spain
}

\section{ABSTRACT}

The effect of goat or cow milk-based diets, with either normal $\mathrm{Fe}$ content or an Fe overload, on bone turnover and the mineralization process was studied in control and anemic rats during chronic Fe repletion. One hundred eighty male Wistar rats were studied during a pre-experimental period of $40 \mathrm{~d}$ in which they were randomly divided into 2 groups, a control group receiving the AIN-93G diet with normal Fe content (45 $\mathrm{mg} / \mathrm{kg}$ of diet) and the Fe-deficient group receiving the AIN-93G diet with low Fe content ( $5 \mathrm{mg} / \mathrm{kg}$ of diet) for $40 \mathrm{~d}$. After the pre-experimental period, the rats were fed for 10,30 , or $50 \mathrm{~d}$ with goat or cow milk-based diets with a normal Fe content $(45 \mathrm{mg} / \mathrm{kg}$ of diet) or an $\mathrm{Fe}$ overload (450 mg/kg of diet). In anemic rats, goat milk with normal Fe content increased levels of the biomarker of bone formation N-terminal propeptides of type I procollagen and diminished parathyroid hormone levels after only $10 \mathrm{~d}$ of supplying this diet, indicating the beginning of restoration of the bone demineralization induced by the anemia, which was not observed with cow milk. After $30 \mathrm{~d}$ of supplying the milk-based diets with normal Fe content or an Fe overload, biomarkers of bone formation and bone resorption were not different between control and anemic rats, indicating that the bone demineralization induced by the Fe-deficiency anemia had recovered, although the process of stabilization of bone turnover began earlier in the animals fed goat milk. In addition, a higher Ca deposit was observed in femur, which positively affects bone mineralization, as well as an increase of Fe in sternum, which indicates that the hematopoietic process essentially recovered earlier on the goat milk diet compared with the cow milk diet.

Key words: goat and cow milk, iron deficiency anemia, iron repletion, bone turnover

Received November 26, 2010.

Accepted February 22, 2011.

${ }^{1}$ Corresponding author: javierdc@ugr.es

\section{INTRODUCTION}

Iron-deficiency anemia is characterized by the reduction or absence of Fe stores, low serum concentrations of Fe and hemoglobin, hematocrit reduction, and increased platelet counts (Campos et al., 1998). Iron overload is a frequent consequence of treatment for anemia and is less common than Fe deficiency (Parkkila et al., 2001). On the other hand, it is not clear why the role of $\mathrm{Fe}$ in bone formation and disease has not received much attention, given the known biochemical role that Fe has upon collagen maturation and the high prevalence of Fe-deficiency anemia (Medeiros et al., 2002).

Previous studies (Campos et al., 1998, 2007) reported that Fe-deficient rats had decreased femur mineralization that was accompanied by higher levels of cortisol and parathyroid hormone (PTH), increasing bone fragility. Moreover, Katsumata et al. (2009) reported that severe Fe deficiency suppressed both bone formation and resorption, facts that encouraged us to extend our study of bone turnover. Some researchers have examined the relationship between dietary $\mathrm{Fe}$ intake and bone metabolism. Medeiros et al. (1997, 2002, 2004) hypothesized that Fe exerts its influence on bone through collagen synthesis. Iron is a required cofactor for prolyl and lysyl hydroxylase enzymes, and this mineral is essential for lysyl oxidase activity, which then catalyzes cross-linking of adjacent collagen fibers. In Fe deficiency less Fe may be available to the prolyl and lysyl hydroxylase enzymes, which could result in decreased cross-linking activity and, subsequently, weaker collagen fibers (Tuderman et al., 1977).

Most of the $\mathrm{Ca}$ in the body is stored in the bone and is found in serum in 2 forms: ionized and ready for use or not ionized and bound to protein (Campos et al., 2007). The maintenance of $\mathrm{Ca}$ concentrations is managed through hormonally coordinated action. Parathyroid hormone increases blood concentrations of $\mathrm{Ca}$, stimulating production of the biologically active form of vitamin $\mathrm{D}_{3}, 1,25$-dihydroxycholecalciferol $[1,25$ $\left.(\mathrm{OH})_{2} \mathrm{D}_{3}\right]$, in the kidney. This hormone favors mobilization of $\mathrm{Ca}$ from bone and maximizes renal tubular reabsorption of Ca. Moreover, vitamin D facilitates Ca 
absorption from the small intestine and enhancing its fluxes out of bone.

Bone is a connective tissue that consists essentially of a mineralized extracellular matrix and specialized cells. The principal organic component of the matrix is the type I collagen, which represents $90 \%$; the remaining $10 \%$ is composed of a series of nonstructural proteins of minor size (Burgeson, 1988). The inorganic phase is composed of minuscule crystals of hydroxyapatite, inserted in the collagen fibers, allowing this form of stored Ca to be rapidly liberated in case of need when intake is inadequate. These elements play a crucial role in numerous physiological functions. The homeostasis of these minerals is regulated by mechanisms that control the formation and resorption of the bone tissue, processes that can be altered by different environmental, nutritional, genetic, and endocrine factors, giving place to abnormal compositions or pathologies of the bone (Ehrlich and Lanyon, 2002).

The interaction existing between $\mathrm{Ca}$ and $\mathrm{Fe}$ is well known. Divalent metal transporter-1, the principal mechanism by which nonheme Fe is taken up at the intestinal brush border, is shared also by $\mathrm{Ca}$. Therefore, $\mathrm{Ca}$ acts as a low-affinity noncompetitive inhibitor (but not a transported substrate) of divalent metal transporter-1, explaining the inhibitory effect of dietary Ca on Fe bioavailability (Shawki and Mackenzie, 2010).

Milk has a high Ca bioavailability and therefore can affect in a positive way the process of bone mineralization (Campos et al., 2007). Goat milk improves Fe bioavailability in anemic rats, increasing Fe deposits in target organs and favoring the recovery of hematological parameters. Goat milk fat is richer in medium-chain triglycerides, which are rapidly absorbed and metabolized to obtain energy increasing the synthesis of carrier proteins and thus the absorption of Fe. Goat milk has higher contents of vitamin $\mathrm{A}$ and $\mathrm{C}$, which would favor once again $\mathrm{Fe}$ absorption (Alférez et al., 2006). In addition, goat milk consumption improves the digestive and metabolic utilization of $\mathrm{Ca}$ and $\mathrm{P}$ and their deposits in the corresponding target organs, minimizing the interactions between Ca and Fe (Campos et al., 2007).

These precedents led us to study the influence of goat or cow milk-based diets, with either a normal $\mathrm{Fe}$ content or Fe overload, and supplied for 10, 30, or 50 $\mathrm{d}$, on the bone remodeling process impaired because of Fe-deficiency anemia, to determine if goat milk consumption could have a positive effect on this process.

\section{MATERIALS AND METHODS}

\section{Animals}

Male Wistar albino rats $(\mathrm{n}=180)$ recently weaned (21 d old), weighing $40 \pm 5 \mathrm{~g}$, from the University of
Granada Laboratory Animal Service (Granada, Spain) were used for this study. Animal care procedures and experimental protocols were approved by the Ethics Committee of the University of Granada in accordance with the European Community guidelines.

\section{Experimental Design and Diets}

After weaning, rats were randomly divided into 2 groups, a control group fed with the AIN-93G diet (Reeves et al., 1993) and an anemic group that received the same diet but with a low $\mathrm{Fe}$ content $(5 \mathrm{mg}$ of $\mathrm{Fe} /$ $\mathrm{kg}$ of diet) during the 40-d pre-experimental period (Pallarés et al., 1993). After induction of anemia (d 0 of the study), the experimental period (EP) began, in which the control and anemic groups were fed for 10, 30 , or 50 d with 4 diets: goat or cow milk-based diet with normal $\mathrm{Fe}$ content $(45 \mathrm{mg} / \mathrm{kg}$ ) or Fe overload (450 $\mathrm{mg} / \mathrm{kg}$ ). The dose of $450 \mathrm{mg}$ of $\mathrm{Fe} / \mathrm{kg}$ is common for inducing chronic Fe overload in animal studies (Raja et al., 1994). The diets included ferric citrate $\left(\mathrm{C}_{6} \mathrm{H}_{5} \mathrm{FeO}_{7}\right)$ as the Fe source.

From the beginning of the study, animals were placed in individual metabolic cages in an environmentally controlled room with a constant temperature of $22^{\circ} \mathrm{C}$ \pm 2 , a 12-h light-dark cycle, and $55 \pm 10 \%$ humidity. Mineral-free water was available ad libitum, but the diet intake was controlled by pair feeding all the animals to avoid any differences due to intake. The diet supplied was calculated as $80 \%$ of the average intake of each experimental period. On d 0 of the study, peripheral blood samples from the caudal vein were collected using EDTA as anticoagulant for hematological analysis from all rats to confirm that Fe-deficiency anemia has been reached. After fasting overnight, 10 animals per group (10 controls and 10 anemic) were weighed and anesthetized by an intraperitoneal injection of $5 \mathrm{mg}$ of sodium pentobarbital (Sigma Diagnostics, St. Louis, MO) per $100 \mathrm{~g}$ of BW. After median laparotomy, the rats were totally bled out by cannulation of the abdominal aorta; the sternum and femur were removed immediately, and stored at $-40^{\circ} \mathrm{C}$ until further analysis of mineral content.

Blood on d 10 was collected from caudal vein, because animals were not culled at this point. On d 30 and 50 animals were anesthetized and totally bled out by cannulation of the abdominal aorta $(n=80$ on $d 30$ and $n$ $=80$ on $\mathrm{d} 50$ ), and femur and sternum were removed. Bone turnover was assessed on d 10, 30, and 50 of the study by measuring N-terminal propeptides of type I procollagen (PINP), C-terminal telopeptides of type I collagen $(\mathbf{C T x})$, tartrate resistant acid phosphatase $5 \mathrm{~b}$ (TRACP 5b), and PTH in serum. Figure 1 shows the experimental design of the study. 


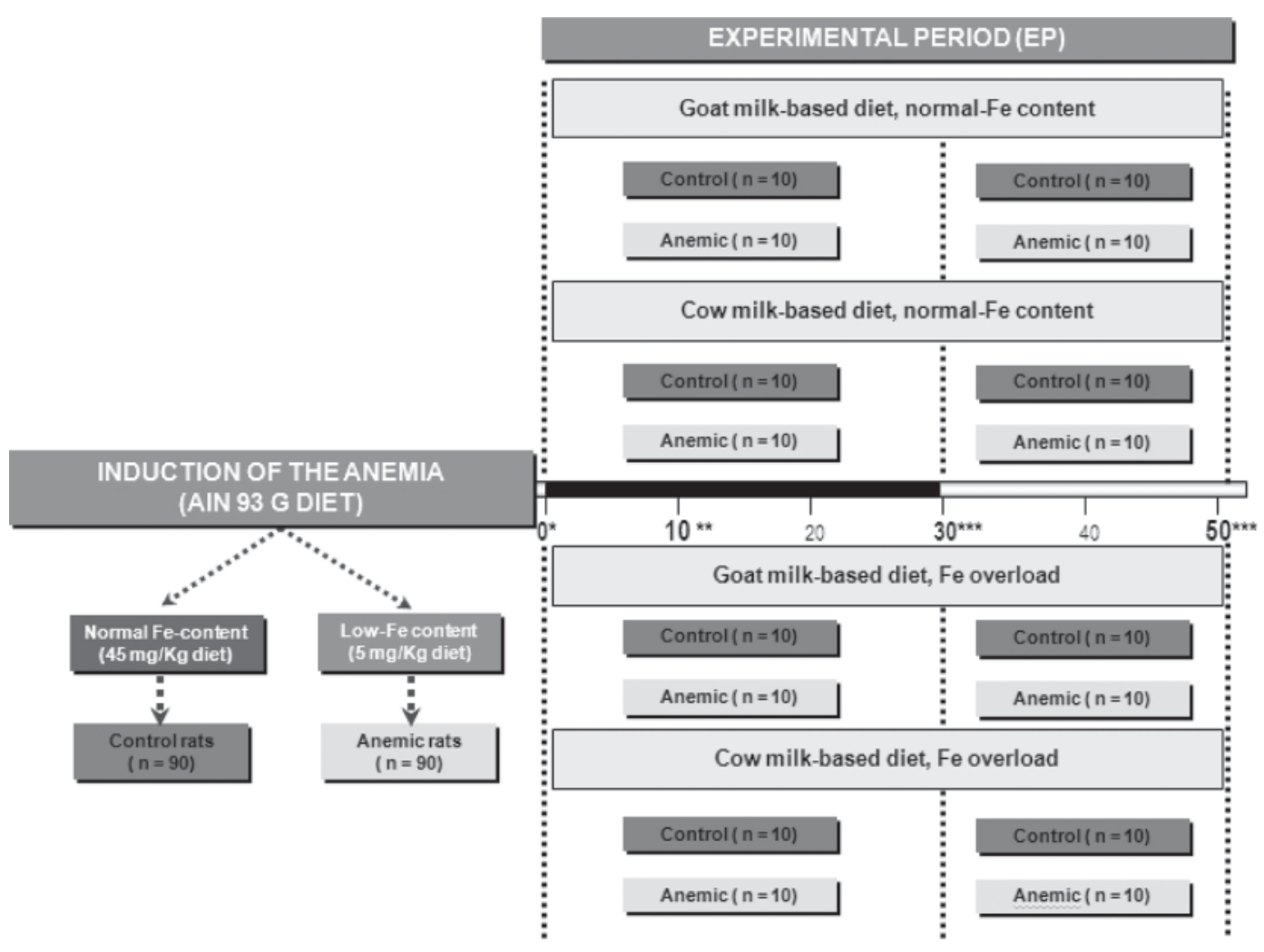

Figure 1. Experimental design of the study. On d $0\left(^{*}\right)$, blood from abdominal aorta (10 animals per group) was withdrawn to measure hematological parameters; femur and sternum were removed for mineralization analysis. On d $10(* *)$, blood samples from the caudal vein were obtained to measure bone turnover parameters. On d 30 and $50(* * *)$, blood from the abdominal aorta was withdrawn to measure bone turnover parameters; femur and sternum were removed for mineralization analysis. AIN = American Institute of Nutrition.

Table 1 summarizes the diets supplied during the EP. The milk-based diets were made with skim cow (Holstein breed) or goat milk (Murciano-Granadina breed) powder. Goat or cow skim milk samples were formulated to provide $20 \%$ of protein in the diets. Because the fat content in the milk was undetectable $(<0.01 \%)$, fat was added to reach a diet with $10 \%$ fat, obtained by purification of skim or butter (from goat or cow milk, respectively). The mineral contents in the normal $\mathrm{Fe}$ diets used during the EP by analysis were $40.5 \mathrm{mg}$ of $\mathrm{Fe} / \mathrm{kg}, 5,136 \mathrm{mg}$ of $\mathrm{Ca} / \mathrm{kg}$, and $3,067 \mathrm{mg}$ of $\mathrm{P} / \mathrm{kg}$ (goat milk-based diet) and $39.6 \mathrm{mg}$ of $\mathrm{Fe} / \mathrm{kg}, 5,146 \mathrm{mg}$ of $\mathrm{Ca} / \mathrm{kg}$, and $3,127 \mathrm{mg}$ of $\mathrm{P} / \mathrm{kg}$ (cow milk-based diet). Mineral content in the Fe overload diets were $476.4 \mathrm{mg}$ of $\mathrm{Fe} / \mathrm{kg}, 5,093 \mathrm{mg}$ of $\mathrm{Ca} / \mathrm{kg}$, and $3,105 \mathrm{mg}$ of $\mathrm{P} / \mathrm{kg}$ (goat milk-based diet) and $482.0 \mathrm{mg}$ of $\mathrm{Fe} / \mathrm{kg}, 5,100 \mathrm{mg}$ of $\mathrm{Ca} / \mathrm{kg}$, and $3,181 \mathrm{mg}$ of $\mathrm{P} / \mathrm{kg}$ (cow milk-based diet).

\section{DM and Determination of Protein}

Water content in the diet, femur, and sternum was determined by drying the material at $105 \pm 2^{\circ} \mathrm{C}$ until the weight remained constant $(\sim 48 \mathrm{~h})$. The nitrogen content in the skim milks and diets was determined by the Kjeldahl method, using a protein conversion factor of 6.38 (Kjeldahl, 1983).

\section{Measurement of $\mathrm{Ca}, \mathrm{P}$, and Fe}

The concentrations of $\mathrm{Ca}$ and $\mathrm{P}$ were measured in femur and Fe was determined in sternum. In addition, the same minerals were measured in the diet. The concentrations of $\mathrm{Ca}$ and $\mathrm{Fe}$ in the diet and bones were determined by atomic absorption spectrophotometry (AAnalyst 1100B spectrometer with WinLab32 for AA software, PerkinElmer, Beverly, MA). The concentration of $\mathrm{P}$ was analyzed by visible spectrophotometry (UV/VIS spectrometer lambda 16, Perkin-Elmer) using the Fiske and Subbarow (1925) technique. The samples had been previously mineralized by wet method in a sand bath (J.R. Selecta, Barcelona, Spain). Samples of skim milk powder and lyophilized bovine liver (certified reference material CRM $063 \mathrm{R}$ and BCR 185; Commission of the European Community Bureau of Reference, Brussels, Belgium), were used simultaneously to test the $\mathrm{Ca}$ (skim milk powder) and Fe recovery (bovine 
Table 1. Composition of the diets supplied during the experimental period

\begin{tabular}{lc}
\hline Diet $^{1}$ & $\begin{array}{c}\text { Amount, } \\
\mathrm{g} / \mathrm{kg}\end{array}$ \\
\hline Goat milk diet (normal Fe or Fe overload) & \\
Goat milk protein & 200 \\
Goat milk lactose & 341 \\
Goat milk fat & 100 \\
Wheat starch & 160 \\
Constant ingredients & \\
& 199 \\
Cow milk diet (normal Fe or Fe overload) & \\
Cow milk protein & \\
Cow milk lactose & 200 \\
Cow milk fat & 353 \\
Wheat starch & 100 \\
Constant ingredients & \\
\end{tabular}

${ }^{1}$ The diets were prepared according to the recommendations of the American Institute of Nutrition (AIN; Reeves et al., 1993) for control (normal $\mathrm{Fe}$ ), and $\mathrm{Fe}$ overload was induced according to recommendations of Raja et al. (1994).

${ }^{2}$ The constant ingredients consisted of $(\mathrm{g} / \mathrm{kg}$ of diet): fiber (micronized cellulose) 50, sucrose 100 , choline chloride 2.5 , L-cystine 1.8 , mineral premix 35 , vitamin premix 10 . The vitamin premix was prepared according to the recommendations of the AIN (Reeves et al., 1993). The mineral premix for goat and cow milk-based diets was formulated taking into account the mineral content of the skim milk powder supplied to meet the recommendations of the AIN (Reeves et al., 1993). All the diets were isocaloric $(17,226 \mathrm{~kJ})$.

liver $)$ : Ca value $=13.88 \pm 0.10 \mathrm{mg} / \mathrm{g} ; \mathrm{Fe}$ value $=210$ $\pm 3 \mu \mathrm{g} / \mathrm{g}$; mean \pm SEM of 5 determinations; certified values: $\mathrm{Ca}=13.49 \pm 0.10 \mathrm{mg} / \mathrm{g} ; \mathrm{Fe}=214 \pm 5 \mu \mathrm{g} / \mathrm{g})$.

\section{Hematological Tests}

Hemoglobin (Hb) concentration, red blood cells (RBC), hematocrit, mean corpuscular volume (MCV), and platelets were measured using an automated hematology analyzer Sysmex K-1000D (Sysmex, Tokyo, Japan).

\section{PINP}

$\mathrm{N}$-Terminal propeptide of type I procollagen is the most specific and sensitive marker of bone formation (Claudon et al., 2008). The concentration of PINP was determined by using a rat/mouse PINP enzyme immunoassay (Immunodiagnostics System Ltd., Boldon, UK) from serum samples. Calibrators, controls, and samples were added to the wells of the microtiter plate followed by PINP labeled with biotin; the plate was incubated for $1 \mathrm{~h}$ at room temperature before being aspirated and washed. Horseradish peroxidase-labeled avidin was added and bound selectively to complexed biotin; following a further wash step, the color was developed using a chromogenic substrate $\left(3,3^{\prime}, 5,5^{\prime}\right.$-tetramethylbenzidine). The absorbance of the stopped reac- tion mixtures was read in a microplate reader (Bio-Rad Laboratories Inc., Hercules, CA) at $450 \mathrm{~nm}$ (reference $650 \mathrm{~nm}$ ) within $30 \mathrm{~min}$ of adding the stop solution. The color intensity developed was inversely proportional to the concentration of PINP.

\section{Degradation Products from CTX}

Type I collagen accounts for more than $90 \%$ of the organic matrix of bone and is synthesized primary in bone (Burgeson, 1988). During renewal of the skeleton, bone matrix is degraded and fragments of type I collagen are released into the circulation. These fragments were measured using a RatLaps enzyme immunoassay (Immunodiagnostics System Ltd.) from serum samples. This assay is based upon the competitive binding of a polyclonal antibody to soluble RatLaps antigens or to immobilized RatLaps antigens. Briefly, the polyclonal antibody is raised against a synthetic peptide having a sequence specific for a part of the C-terminal telopeptide $\alpha-1$ chain of rat type I collagen. For standardization of the kit, a synthetic peptide specific for the C-terminal telopeptide $\alpha-1$ chain of type I collagen in rats was used. During the preincubation step, biotinylated peptide was immobilized by binding to the streptavidin-coated microtiter wells. The wells were emptied and washed. Standards, controls, and samples (rat serum) were pipetted into appropriate wells, followed by a solution of a primary antibody (polyclonal rabbit). Following the primary incubation step, the wells were emptied and washed. In the secondary incubation step, a solution of a goat anti-rabbit antibody conjugated with peroxidase (secondary antibody) was added and bound to the polyclonal rabbit antibody. After the third washing step, a chromogenic substrate $\left(3,3^{\prime}, 5,5^{\prime}\right.$-tetramethylbenzidine) was added and the color reaction was stopped with $0.18 \mathrm{M} \mathrm{H}_{2} \mathrm{SO}_{4}$. Finally, the absorbance was read using a Bio-Rad microplate reader. The absorbance level is inversely related to concentration of RatLaps antigens in the sample.

\section{TRACP $5 b$}

The concentration of TRACP $5 \mathrm{~b}$ was measured in rat serum using the RatTRAP assay (Immunodiagnostics System Ltd.), which uses a highly characterized, specific monoclonal antibody prepared using baculovirusgenerated recombinant rat TRACP as antigen (Kaija et al., 1999). The monoclonal antibody is incubated in anti-mouse IgG-coated microtiter wells. After washing, standard, control, and serum samples were incubated in the wells, and bound TRACP 5b activity was determined with a chromogenic substrate to develop color. The reaction was stopped and the absorbance of the 
reaction mixture was read in a microplate reader (BioRad Laboratories Inc.) at $405 \mathrm{~nm}$ within $30 \mathrm{~min}$ of adding the stop solution. Color intensity was directly proportional to the activity of TRACP $5 \mathrm{~b}$ present in the sample. Because secreted TRACP $5 \mathrm{~b}$ is indicative of the number of osteoclasts, these values can be used to replace histological determination of osteoclast number in rat bone (Alatalo et al., 2004; Rissanen et al., 2006).

\section{PTH}

Serum PTH was measured using a rat PTH immunoradiometric assay kit (ALPCO Diagnostics, Salem, MA). Two different goat antibodies to the N-terminal region (1-34) of rat PTH were purified by affinity chromatography. One of the antibodies was immobilized onto plastic beads to capture the PTH molecules and the other antibody was radiolabeled for detection. Serum samples were incubated simultaneously with an antibody-coated bead and the ${ }^{125}$ I-labeled antibody. Both intact PTH (1-84) and N-terminal PTH (1-34) contained in the sample were immunologically bound by the immobilized antibody and the radiolabeled antibody to form a "sandwich" complex. At the end of the incubation period, the beads were washed to remove any unbound labeled antibody and other components. The radioactivity bound to the beads was then measured in a Wizard Automatic Gamma Counter (Perkin Elmer, Waltham, MA). The radioactivity of the antibody complex bound to the bead was directly proportional to the amount of rat PTH in the sample. A standard curve was generated by plotting the counts per minute against the respective rat PTH concentration for each standard on logarithmic scales. The concentration of PTH in the samples was determined directly from this curve.

\section{Statistical Analysis}

Data are reported as means \pm SEM. Differences between groups (control vs. anemic and normal Fe vs. Fe overload) were tested for statistical significance with the Student's $t$-test. One-way ANOVA was used to compare the different diets supplied to the 2 groups (control and anemic) during EP. Individual means were tested by pair-wise comparison using Tukey's post hoc multiple comparison test when main effects and interactions were significant. Data were analyzed by 2 -way ANOVA to determine the effects of anemia, type of diet, and Fe content in the diet. A level of $P<0.05$ was considered to indicate statistical significance. The SPSS software package, version 15.0 (2008, SPSS Inc., Chicago, IL) was used for data treatment and statistical analysis.

\section{RESULTS AND DISCUSSION}

At d 0, all hematological parameters in the anemic rats (that consumed the low-Fe diet) differed from those of the control rats. Most of the hematological parameters were lower: serum Fe $(1,372 \pm 121 \mu \mathrm{g} / \mathrm{L}$ in control group vs. $710 \pm 58 \mu \mathrm{g} / \mathrm{L}$ in anemic group; $P<$ $0.001), \mathrm{Hb}$ concentration $(124.3 \pm 2.5 \mathrm{~g} / \mathrm{L}$ in control vs.74. $\pm 2.4 \mathrm{~g} / \mathrm{L}$ in anemic, $P<0.001)$, RBC $(7.1 \pm$ $0.15 \times 10^{12} / \mathrm{L}$ in control vs. $6.3 \pm 0.2 \times 10^{12} / \mathrm{L}$ in anemic; $P<0.001)$, hematocrit $(39.5 \pm 0.7 \%$ in control vs. $26.9 \pm 0.4 \%$ in anemic; $P<0.001), \operatorname{MCV}(55.7 \pm 0.3$ $\mathrm{fL}$ in control vs. $40.0 \pm 0.6 \mathrm{fL}$ in anemic; $P<0.001)$, whereas platelets were higher $\left(732 \pm 25.5 \times 10^{9} / \mathrm{L}\right.$ in control vs. $1,389 \pm 66.1 \times 10^{9} / \mathrm{L}$ in anemic; $P<0.001$ ) All these findings were expected and consistent with long-term induced Fe deficiency. The impaired hematological parameters recovered to baseline levels after feeding rats the milk-based diets for 30 or $50 \mathrm{~d}$, as expected with consumption of normal or high-Fe diets (data not shown).

\section{Mineral Content in Femur and Sternum at d 0}

At the beginning of the study (d 0$)$, evaluation of bone mineralization revealed that $\mathrm{Ca}$ and $\mathrm{P}$ contents in femur and Fe deposits in sternum were markedly lower for the anemic group $(P<0.001)$, which was in agreement with previous results (Campos et al., 2007), revealing a high degree of bone demineralization induced by the anemia. Medeiros et al. $(1997,2002,2004)$ reported that Fe deficiency had a significant effect upon bone mineral density, content, and fragility in young rats fed an $\mathrm{Fe}-$ and Ca-restrictive diet. Hydroxylase enzymes require both ascorbate and Fe to function properly. Iron deficiency could lead to decreased hydroxylation of 1,25-dihydroxylcholcalciferol, the active form of vitamin $\mathrm{D}$, resulting in diminished Ca absorption, because the 25-hydroxycholcalciferol-1- hydroxylase is a 3-component system: a flavoprotein, an Fe-sulfur protein, and a cytochrome P-450 (DeLuca, 1976). A lack of Fe could affect this enzyme system, leading to decreased renal reabsorption of $\mathrm{Ca}$ as well as decreased intestinal $\mathrm{Ca}$ absorption when Fe is limiting (Medeiros et al., 2004). On the other hand, as expected for consumption of a diet with low Fe content, a decrease was observed in the sternum Fe content of anemic rats $(P<0.001)$, which adversely influences the hematopoietic process in this group of animals and confirms the severe degree of $\mathrm{Fe}$ deficiency (Table 2).

\section{Bone Turnover Biomarkers at d 10}

On d 10 of the study, some important changes in bone metabolism were observed, after supplying milk-based 
Table 2. Mineral content in femur and sternum of control and anemic rats on $\mathrm{d} 0$ of the study ${ }^{1}$

\begin{tabular}{lcr}
\hline Mineral content & $\begin{array}{c}\text { Normal Fe } \\
\text { (control group) }\end{array}$ & $\begin{array}{c}\text { Low Fe } \\
\text { (anemic group) }\end{array}$ \\
\hline Ca in femur, mg/g of DM & $167.44 \pm 3.61$ & $98.76 \pm 3.58^{*}$ \\
$\mathrm{P}$ in femur, mg/g of DM & $124.75 \pm 2.67$ & $101.54 \pm 2.62^{*}$ \\
Fe in sternum, $\mu \mathrm{g} / \mathrm{g}$ of DM & $78.53 \pm 4.24$ & $62.05 \pm 2.83^{*}$ \\
\hline
\end{tabular}

${ }^{1}$ Data are means $\pm \mathrm{SE}$ of 10 animals.

*Significantly different $(P<0.001)$ from the control group by Student's $t$-test.

diets with normal Fe content or Fe overload (Table 3). The concentration of PINP increased in the anemic group compared with controls when a goat milk-based diet with normal Fe content was supplied $(P<0.05)$ and in these animals compared with those fed cow milk-based diet $(P<0.001)$. The beneficial effect of goat milk on mineral metabolism has been well documented: it improves Fe bioavailability (Alférez et al., 2006; Nestares et al., 2008), and the Fe is available for hydroxylation processes and then in the biosynthesis of vitamin D. It is known that 25-hydroxycholcalciferol-1hydroxylase is located within the renal mitochondria and this enzyme is Fe-dependent (DeLuca, 1976). Moreover, Fe exerts its influence on bone turnover by affecting type I collagen synthesis and maturation. Iron is an essential cofactor for prolyl and lysyl hydroxylases, enzymes that catalyze ascorbate-dependent hydroxylation of prolyl and lysyl residues, essential steps before crosslinking by lysyl oxidase (Tuderman et al., 1977). Therefore, an improvement in Fe bioavailability would contribute to increase cross-linking activity and, subsequently, stronger collagen fibers. This fact supports the increase in PINP found in animals fed with goat milk-based diets, reflecting the higher amount of bone matrix developed (Cons-Molina, 2003).

Serum levels of bone resorption biomarkers were lower in anemic animals fed a goat milk-based diet with $\mathrm{Fe}$ overload compared with cow milk- based diet $(P<0.01$ for CTx and $P<0.001$ for TRACP 5b), so the increase in the resorption process induced by the anemia seemed to stop in this point following the consumption of goat milk, because the bone remodeling process maintains skeletal mass practically invariable. The increase in TRACP 5b in control and anemic animals fed a cow milk-based diet with $\mathrm{Fe}$ overload compared with those fed a goat milk-based diet $(P<0.001)$ revealed an active process of bone resorption. In this context, Irie et al. (2000) proposed a functional correlation of the TRACP 5b activity in osteocytes with osteocytic osteolysis. In osteoclasts, TRACP $5 \mathrm{~b}$ has been suggested to generate free radicals and to directly participate in bone resorption (Hayman and Cox 1994), to dephosphorylate osteopontin and bone sialoprotein, and to serve as an osteoclast detachment factor (Ek-Rylander et al., 1994). The osteoclasts are mobilized toward the zone of destruction and then adhere tightly to the bone surface, beginning the process of resorption. This negative effect in the process of bone turnover was not observed when the rats consumed goat milk-based diets with $\mathrm{Fe}$ overload, indicating once again a positive effect in the process of recovery of bone demineralization induced by

Table 3. Bone turnover biomarkers at d 10 of the study in control and anemic groups fed with goat- or cow-based diets with normal Fe content or Fe overload ${ }^{1}$

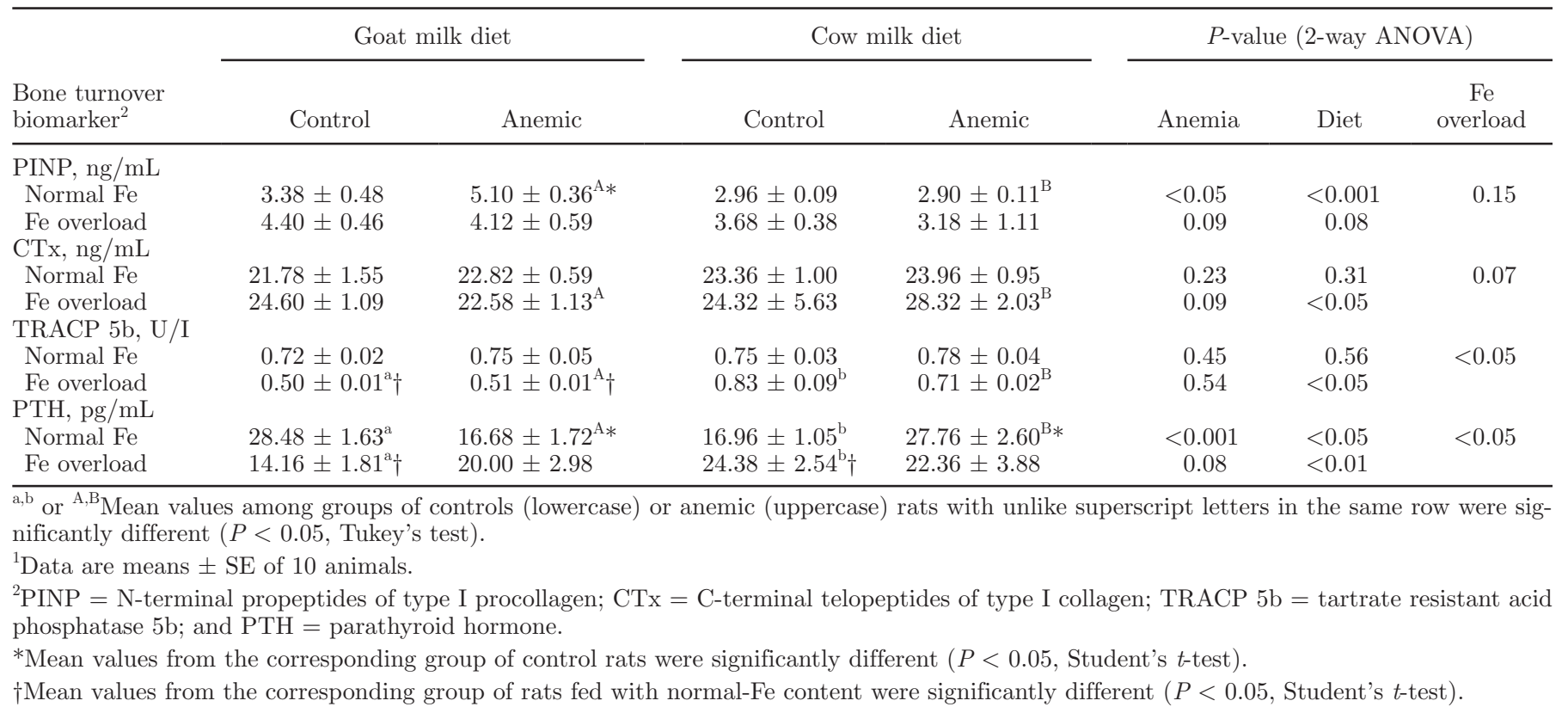


the Fe deficiency. In general, the results showing that osteoclastic activity (TRACP 5b) and, therefore, bone resorption were lower indicate that $\mathrm{Fe}$ overload in the diet for $10 \mathrm{~d}$ induced stabilization of bone turnover in anemic animals. This first experimental period (10 d of consumption of diets with normal or high Fe content) can be considered transitional, emphasizing that, in the animals fed goat milk-based diets, the process of bone remodeling had already begun to stabilize, although at the view of the short time of supply of the diets, a certain imbalance in the studied biomarkers was observed.

With regard to PTH levels, no significant differences caused by anemia were found in the groups that consumed either milk-based diet with $\mathrm{Fe}$ overload for $10 \mathrm{~d}$, whereas feeding diets with normal Fe content resulted in a decrease of PTH in the anemic animals that consumed goat milk $(P<0.001)$ and an increase in those that consumed cow milk $(P<0.001)$. Iron overload produced a marked decrease of PTH levels in control animals that consumed goat milk-based $\operatorname{diet}(P$ $<0.001)$, whereas an increase was observed $(P<0.05)$ in those fed a cow milk-based diet. These results are in agreement with the increase in nutritive utilization of $\mathrm{Ca}$, because of the greater absorption of this mineral, as previously reported Campos et al. (2007).

\section{Bone Turnover Biomarkers at d $\mathbf{3 0}$ and 50}

At d 30 of the study, no effect of anemia was observed for any of the diets as assayed by the biomarker of bone formation PINP or by the resorption biomarkers CTx and TRACP 5b, revealing that the bone demineralization induced by the lack of Fe was restored. The process of stabilization of turnover began earlier in the animals fed goat milk, as indicated by PINP levels only $10 \mathrm{~d}$ into the EP. The supply of diets extended for 50 $\mathrm{d}$ caused diminished CTx and TRACP 5b in anemic animals that received the goat milk diet with normal Fe content $(P<0.001$; Table 4$)$. The decrease of these resorption biomarkers in anemic rats at d 50 confirmed that the remodeling process was normalized after supplying goat milk, probably because goat milk minimizes the interactions between $\mathrm{Fe}$ and $\mathrm{Ca}$ (Barrionuevo et al., 2002; Campos et al., 2003). Moreover, as it shows the evolution of PINP, the bone formation is lower at 50 $\mathrm{d}$ of the study in comparison with the previous periods (10 and $30 \mathrm{~d}$ ), because at this point the rats were evolved to the adult stage (McCutcheon and Marinelli, 2009), the active growth had finished and remodeling process had become stable.

In the anemic rats that consumed the goat milk-based diet with normal Fe content for $30 \mathrm{~d}$, a marked decrease in PTH levels occurred compared with controls $(P<$ 0.001; Table 4). This decrease in PTH levels was prob- ably due to the increase in the nutritive utilization of $\mathrm{Ca}$, following major absorption of this mineral (Campos et al., 2007). Nevertheless, for the cow milk-based diet with $\mathrm{Fe}$ overload, the levels of this hormone increased in anemic animals $(P<0.05)$, which could be due to the decrease in the serum levels of ionic Ca in situations of Fe deficiency (Masuyama et al., 2000). By effect of Fe overload, at d 30 and 50 of the study, PTH levels increased in all the experimental groups $(P<0.001$; Table 4). This finding might be because PTH increases the speed of bone remodeling and the mobilization of Ca deposit in the organism, which is in agreement with Pawlotsky et al. (1999) and Dimitriadou et al. (2010), who found an increase in the serum levels of PTH in Fe overload conditions. After $50 \mathrm{~d}$ of feeding milk-based diets, PTH levels were not modified by the effect of anemia or type of diet in rats fed normal Fe diets, indicating that the speed of bone remodeling had decreased because of the complete recovery of bone metabolism.

According to Hale et al. (2007), PINP levels can be correlated directly to PTH levels, and recent studies reveal that at intermittent doses and within the physiological range, PTH favors bone formation through an increase of the number of osteoblasts (Cosman and Lindsay, 2004), because PTH stimulates the transformation of osteocytes in osteoblasts (Langub et al., 2001) and diminishes the apoptosis of these cells (Bringhurst, 2002).

\section{Mineral Content in Femur and Sternum at $d \mathbf{3 0}$ and 50}

In relation to $\mathrm{Ca}$ deposits in femur, after supplying the diets with normal or high Fe content for 30 or $50 \mathrm{~d}$ (Table 5), no effect of anemia was observed in animals that consumed goat milk-based diets. However, at d 30, Ca femur content was lower in anemic rats fed cow milk-based diets with normal Fe content $(P<0.01)$, and at $\mathrm{d} 50$ in rats fed cow-milk-based diets with normal and high Fe contents $(P<0.01)$. Deposit of $\mathrm{P}$ in femur was not affected by anemia after 30 or $50 \mathrm{~d}$. By effect of the diet, Ca deposits in femur increased in controls $(P<0.001)$ and anemic rats $(P<0.001)$ fed goat milk with normal or high Fe content at d 30 and 50 , and also $\mathrm{P}$ deposits increased in controls $(P<0.01)$ and anemic rats $(P<0.001)$ that consumed goat milkbased diets with normal Fe content (Table 5).

Goat milk has a high content of vitamin D (Alférez et al., 2006), which favors Ca energy-dependent transcellular saturable transport. Moreover, the lactose in this type of milk is more digestible compared with cow milk and easy to absorb. According to Heaney (1996), these factors favor Ca absorption via paracellular pathway, which explains the greater Ca deposits in the femurs of 
Table 4. Bone turnover biomarkers at d 30 or 50 of the study in control and anemic groups fed with goat- or cow-based diets with normal Fe content or Fe overload ${ }^{1}$

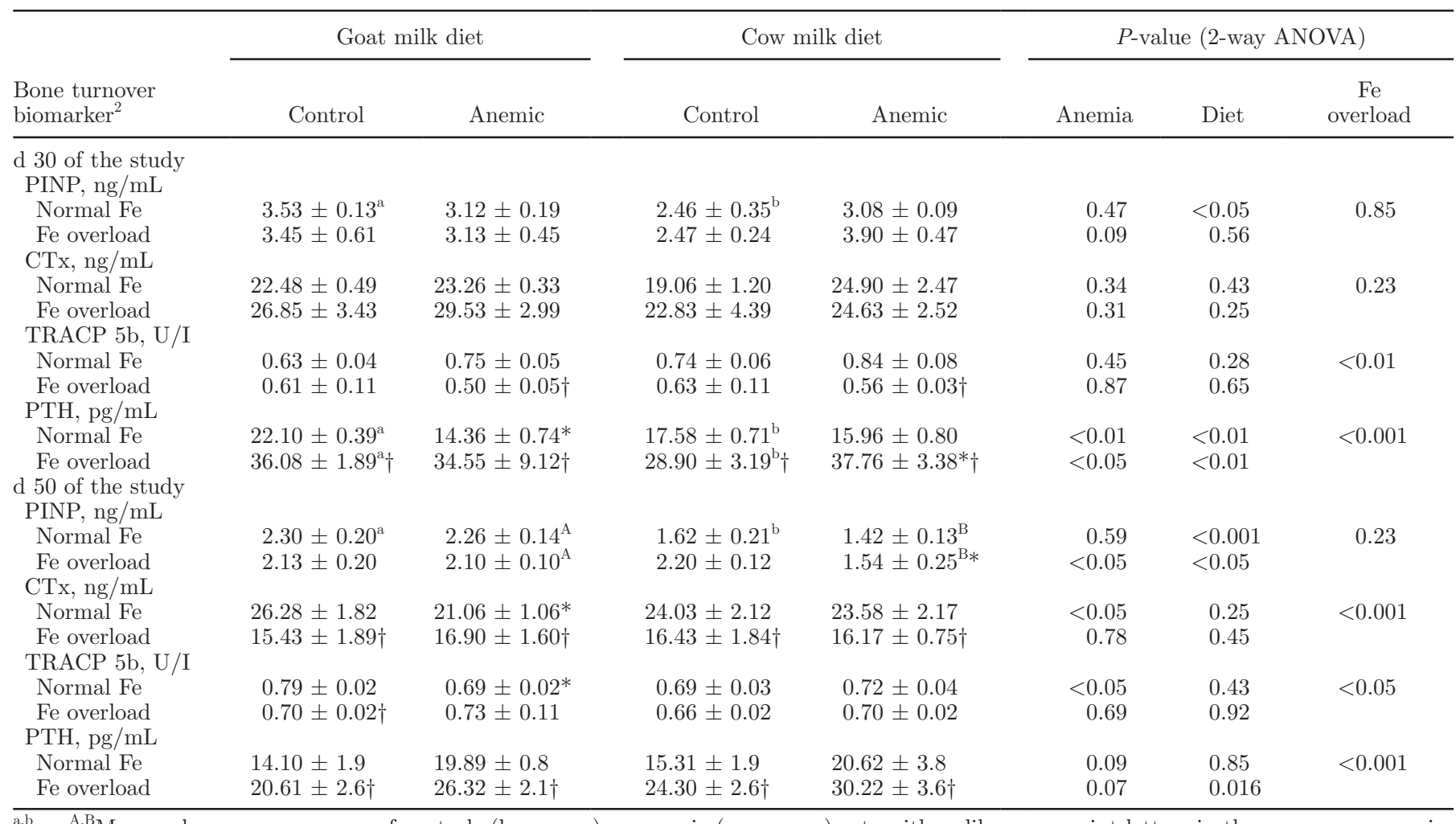

$\overline{\mathrm{a}, \mathrm{b}}$ or ${ }^{\mathrm{A}, \mathrm{B}}$ Mean values among groups of controls (lowercase) or anemic (uppercase) rats with unlike superscript letters in the same row were significantly different $(P<0.05$, Tukey's test $)$.

${ }^{1}$ Data are means $\pm \mathrm{SE}$ of 10 animals.

${ }^{2} \mathrm{PINP}=\mathrm{N}$-terminal propeptides of type I procollagen; CTx $=$ C-terminal telopeptides of type I collagen; TRACP $5 \mathrm{~b}=$ tartrate resistant acid phosphatase 5b; and PTH = parathyroid hormone.

*Mean values from the corresponding group of control rats were significantly different $(P<0.05$, Student's $t$-test).

$\dagger$ Mean values from the corresponding group of rats fed with normal-Fe content were significantly different $(P<0.05$, Student's $t$-test $)$.

rats fed goat milk diets with normal Fe or Fe overload. This could be because nutritive utilization of Fe from goat milk is better than from cow milk, thereby replenishing more efficiently the depleted body stores and, under these conditions, the quantity of available oxygen and ATP increases, recovering the carriers of Ca most efficiently, which favors $\mathrm{Ca}$ absorption via active transport (Conrad and Umbreit, 1993). Something similar happens with $\mathrm{P}$, because the absorption of this element is performed, in part, via active transport (López-Aliaga et al., 1994). In addition, goat milk reduces the interactions between $\mathrm{Ca}$ and $\mathrm{Fe}$, thus increasing the digestive utilization of both minerals (Barrionuevo et al., 2002; Campos et al., 2003). The lower interaction between Ca and $\mathrm{Fe}$ in animals fed goat milk diet can improve $\mathrm{Ca}$ nutritive utilization (Barrionuevo et al., 2002; Campos et al., 2003; Alférez et al., 2006). The higher Ca and P deposits in femur favor the formation of the inorganic component of the bone, because $\mathrm{Ca}$ and $\mathrm{P}$ are the principal constituents of the hydroxyapatite crystals that inlay in the collagen fibers to form a material that has the suitable characteristics of hardness, flexibility, and resistance to constitute the inorganic phase of bone tissue.

After 30 and $50 \mathrm{~d}$ of feeding diets with Fe overload, lower Ca deposits in femur were observed in all experimental groups $(P<0.01$ for control rats and $P<0.001$ for anemic rats); $\mathrm{P}$ deposits showed the same tendency, although the differences were not significant. These results can be because PTH levels were increased with the Fe overload, which is in accordance with Pawlotsky et al. (1999) and Dimitriadou et al. (2010), and this hormone increases the mobilization of $\mathrm{Ca}$ and $\mathrm{P}$ bone deposit.

By the effect of anemia, Fe deposits in sternum were lower at d 30 in the animals that consumed goat milkbased diet with normal Fe content $(P<0.05)$, whereas in animals fed cow milk diet with normal Fe content this decrease in sternum Fe deposits was observed at d 30 and $50(P<0.001)$. Therefore, it is evident that 
Table 5. Mineral content in bone tissues at d 30 and 50 of the study in control and anemic rats fed goat or cow milk-based diets with normal Fe content or Fe overload ${ }^{1}$

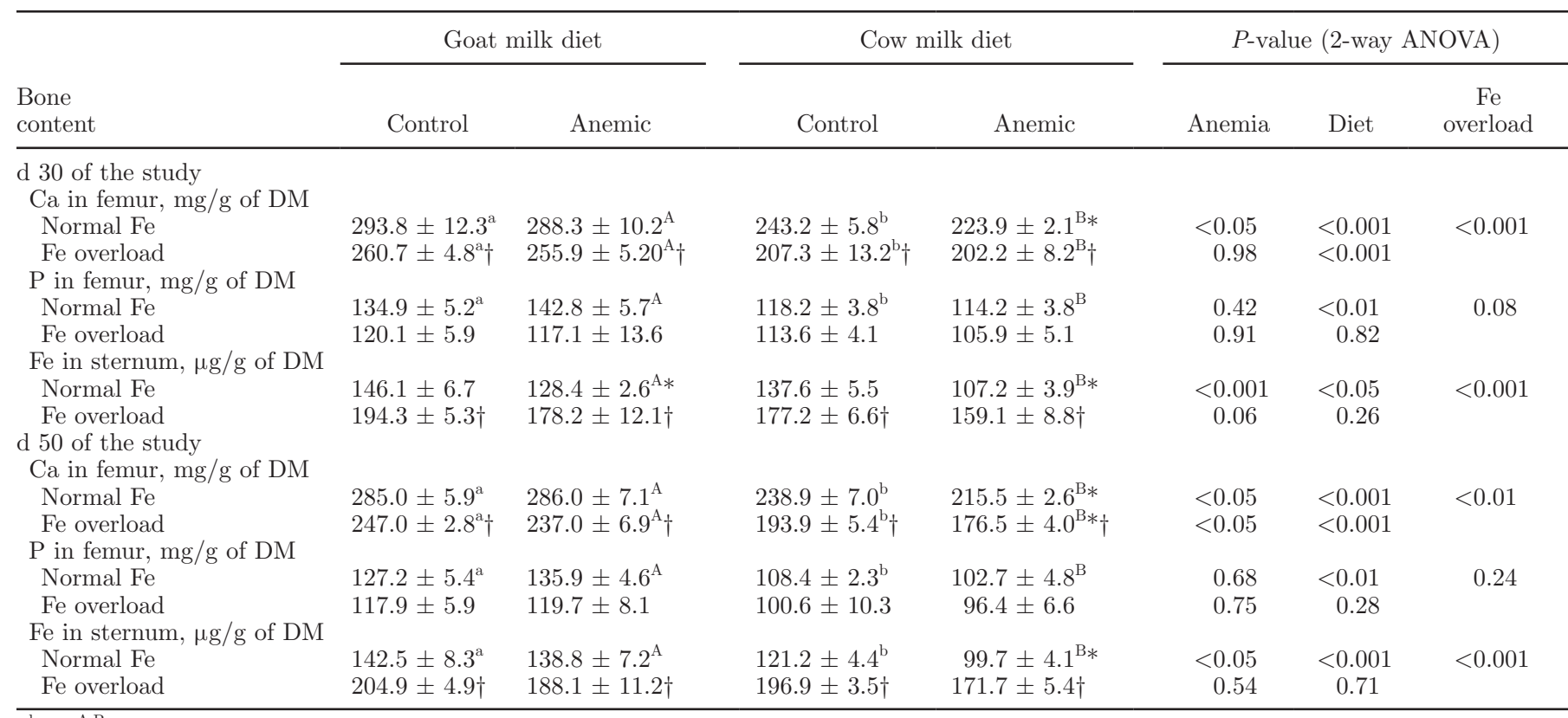

$\overline{\mathrm{a}, \mathrm{b}}$ or ${ }^{\mathrm{A}, \mathrm{B}}$ Mean values among groups of controls (lowercase) or anemic (uppercase) rats with unlike superscript letters in the same row were significantly different $(P<0.05$, Tukey's test).

${ }^{1}$ Data are means $\pm \mathrm{SE}$ of 10 animals.

*Mean values from the corresponding group of control rats were significantly different $(P<0.05$, Student's $t$-test $)$.

$\dagger$ Mean values from the corresponding group of rats fed with normal-Fe content were significantly different $(P<0.05$, Student's $t$-test).

the repletion of Fe in sternum had begun earlier in rats fed the goat milk diet and it is noteworthy that this bone has an important hematopoietic function. If the incorporation of $\mathrm{Fe}$ in the synthesis of hemoglobin is fundamental in the process of development of erythroblasts to reticulocytes, it is at this stage of cell differentiation when the lack of $\mathrm{Fe}$ can damage the complete hemoglobinization of the erythrocytes, giving place to a functional Fe deficiency (Besarab et al., 2009). Iron overload increased $\mathrm{Fe}$ deposit in sternum in both periods in all the experimental groups $(P<0.001)$, as expected. After supplying the goat milk-based diet with normal $\mathrm{Fe}$ content for 30 or $50 \mathrm{~d}$, the Fe deposit in sternum increased in anemic animals compared with animals fed cow milk-based diet $(P<0.001)$. Therefore, in view of the evolution of the concentration of $\mathrm{Fe}$ in sternum, it is possible to affirm that the hematopoietic process, altered as consequence of the Fe restriction to induce the Fe-deficiency anemia, was restored $50 \mathrm{~d}$ after supplying goat milk diet, whereas a longer time was necessary with the cow milk diet.

\section{CONCLUSIONS}

In only $10 \mathrm{~d}$ of supplying goat milk, bone demineralization induced by Fe deficiency began to recover, as evidenced by the increase of the bone formation biomarker PINP and diminishing PTH levels. If the consumption of milk-based diets was prolonged (for 30 or $50 \mathrm{~d}$ ), the parameters of bone remodeling recovered with both milk-based diets, although bone remodeling was restored earlier in animals fed the goat milk-based diets, and feeding a goat milk-based diet favors the mineralization of femur and sternum, improving bone metabolism and hematopoietic process. Therefore, inclusion of goat milk is recommended in diets of humans who suffer nutritional Fe-deficiency anemia, because this type of milk helps to relieve the adverse effects produced in bone turnover due to the Fe deficiency.

\section{ACKNOWLEDGMENTS}

The authors are grateful to Lactalis Iberia S.A. (Lugo, Spain) for providing the dairy products used in this study, to the University of Granada for the personal support of J. Díaz-Castro, and to Elisa Alcover University of Granada, Spain) for her efficient administrative support. This work was supported by CICYT research project no. AGL-2006-02301/ALI.

\section{REFERENCES}

Alatalo, S. L., K. K. Ivaska, S. G. Waguespack, M. J. Econs, H. K. Väänänen, and J. M. Halleen. 2004. Osteoclast-derived serum tartrate-resistant acid phosphatase $5 \mathrm{~b}$ in Albers-Schonberg dis- 
ease (type II autosomal dominant osteopetrosis). Clin. Chem. 50:883-890.

Alférez, M. J. M., I. López-Aliaga, T. Nestares, J. Díaz-Castro, M. Barrionuevo, P. B. Ros, and M. S. Campos. 2006. Dietary goat milk improves iron bioavailability in rats with induced nutritional ferropenic anaemia in comparison with cow milk. Int. Dairy J. $16: 813-821$.

Barrionuevo, M., M. J. M. Alférez, I. López-Aliaga, M. R. Sanz Sampelayo, and M. S. Campos. 2002. Beneficial effect of goat milk on nutritive utilization of iron and copper in malabsorption syndrome. J. Dairy Sci. 85:657-664.

Besarab, A., W. H. Hörl, and D. Silverberg. 2009. Iron metabolism, iron deficiency, thrombocytosis, and the cardiorenal anemia syndrome. Oncologist 14:22-33.

Bringhurst, F. R. 2002. PTH receptors and apoptosis in osteocytes. J. Musculoskelet. Neuronal Interact. 2:245-251.

Burgeson, R. E. 1988. New collagens, new concepts. Annu. Rev. Cell Biol. 4:551-577.

Campos, M. S., M. Barrionuevo, M. J. M. Alférez, A. E. Gómez-Ayala, M. C. Rodríguez-Matas, I. López-Aliaga, and F. Lisbona. 1998. Interactions among iron, calcium, phosphorus and magnesium in the nutritionally iron-deficient rat. Exp. Physiol. 83:771-781.

Campos, M. S., M. Barrionuevo, M. J. M. Alférez, T. Nestares, J. Díaz-Castro, P. B. Ros, E. Ortega, and I. López-Aliaga. 2007. Consumption of caprine milk improves metabolism of calcium and phosphorus in rats with nutritional ferropenic anaemia. Int. Dairy J. $17: 412-419$.

Campos, M. S., I. López-Aliaga, M. J. M. Alférez, T. Nestares, and M. Barrionuevo. 2003. Effects of goats' or cows' milks on nutritive utilization of calcium and phosphorus in rats with intestinal resection. Br. J. Nutr. 90:61-67.

Claudon, A., P. Vergnaud, C. Valverde, A. Mayr, U. Klause, and P. Garnero. 2008. New automated multiplex assay for bone turnover markers in osteoporosis. Clin. Chem. 54:1554-1563.

Conrad, M. E., and J. N. Umbreit. 1993. A concise review: Iron absorption. The mucin- mobilferrin-integrin pathway. A competitive pathway for metal absorption. Am. J. Hematol. 42:67-73.

Cons-Molina, F. 2003. Marcadores bioquímicos de remodelado óseo. Rev. Metab. Oseo Min. 1:91-98.

Cosman, F., and R. Lindsay. 2004. Therapeutic potential of parathyroid hormone. Curr. Osteoporos. Rep. 2:5-11.

DeLuca, H. F. 1976. Metabolism of vitamin D: Current status. Am. J. Clin. Nutr. 29:1258-1270.

Dimitriadou, M., A. Christoforidis, M. Economou, I. Tsatra, E. Vlachaki, L. Fidani, G. Katzos, and M. Athanassiou-Metaxa. 2010. Elevated serum parathormone levels are associated with myocardial iron overload in patients with b-thalassaemia major. Eur. J. Haematol. 84:64-71.

Ehrlich, P. J., and L. E. Lanyon. 2002. Mechanical strain and bone cell function: A review. Osteoporos. Int. 13:688-700.

Ek-Rylander, B., M. Flores, M. Wendel, D. Heinegard, and G. Andersson. 1994. Dephosphorylation of osteopontin and bone sialoprotein by osteoclastic tartrate-resistant acid phosphatase. Modulation of osteoclast adhesion in vitro. J. Biol. Chem. 269:14853-14856.

Fiske, C. H., and Y. Subbarow. 1925. The colorimetric determination of phosphorus. J. Biol. Chem. 66:375-400.

Hale, L. V., R. J. Sells Galvin, J. Risteli, Y. L. Ma, A. K. Harvey, X. Yang, R. L. Cain, Q. Zeng, C. A. Frolik, M. Sato, A. L. Schmidt, and A. G. Geiser. 2007. PINP: A serum biomarker of bone formation in the rat. Bone 40:1103-1109.

Hayman, A. R., and T. M. Cox. 1994. Purple acid phosphatase of the human macrophage and osteoclast. Characterization, molecular properties, and crystallization of the recombinant di-iron-oxo protein secreted by baculovirus-infected insect cells. J. Biol. Chem. 269:1294-1300.

Heaney, R. P. 1996. Calcium. Pages 1007-1018 in Principles of Bone Biology. J. P. Bilezikian, G. A. Raisz, and G. A. Rodan, ed. Academic Press, New York, NY.

Irie, K., H. Ozawa, and T. Yajima. 2000. The histochemical and cytochemical changes from formative to resorptive osteocytes. Acta Histochem. Cytochem. 33:385-391.
Kaija, H., J. Jia, Y. Lindqvist, G. Andersson, and P. Vihko. 1999. Tartrate-resistant bone acid phosphatase: Large scale purification of the recombinant enzyme, characterization and crystallization. J. Bone Miner. Res. 14:424-430.

Katsumata, S., R. Katsumata-Tsuboi, M. Uehara, and K. Suzuki. 2009. Severe iron deficiency was reported to suppress both bone formation and resorption. J. Nutr. 139:238-243.

Kjeldahl, J. 1883. A new method for the determination of nitrogen in organic matter. Z. Anal. Chem. 22:366.

Langub, M. C., M. C. Monier-Faugere, Q. Qi, Z. Geng, N. J. Koszewski, and H. H. Malluche. 2001. Parathyroid hormone/parathyroid hormone-related peptide type 1 receptor in human bone. J. Bone Miner. Res. 16:448-456.

López-Aliaga, I., M. J. M. Alférez, F. Lisbona, M. Barrionuevo, S. Hartiti, A. E. Gómez-Ayala, and M. S. Campos. 1994. Influence of vitamin D3 and type of dietary fat on phosphorus absorption in rats with intestinal resection. Nutr. Res. 14:47-57.

Masuyama, R., M. Uehara, and K. Suzuki. 2000. High P diet induces acute secretion parathyroid hormone without alteration of serum calcium levels in rats. Biosci. Biotechnol. Biochem. 64:2316-2319.

McCutcheon, J. E., and M. Marinelli. 2009. Age matters. Eur. J. Neurosci. 29:997-1014.

Medeiros, D. M., J. Ilich, J. Ireton, V. Matkovic, L. Shiry, and R. Wildman. 1997. Femurs from rats fed diets deficient in copper or iron have decreased mechanical strength and altered mineral composition. J. Trace Elem. Exp. Med. 10:197-203.

Medeiros, D. M., A. Plattner, D. Jennings, and B. Stoecker. 2002 Bone morphology, strength, and density are compromised in irondeficient rats and exacerbated by calcium restriction. J. Nutr. 132:3135-3141.

Medeiros, D. M., B. Stoecker, A. Plattner, D. Jennings, and M. Haub. 2004. Iron deficiency negatively affects vertebrae and femurs of rats independently of energy intake and body weight. J. Nutr. 134:3061-3067.

Nestares, T., J. Díaz-Castro, M. J. M. Alférez, I. López Aliaga, M. Barrionuevo, and M. S. Campos. 2008. Calcium-enriched goat milk, in comparison with similarly enriched cow milk, favours magnesium bioavailability in rats with nutritional ferropenic anaemia. J. Sci. Food Agric. 88:319-327.

Pallarés, I., F. Lisbona, I. López-Aliaga, M. Barrionuevo, M. J. M. Alférez, and M. S. Campos. 1993. Effects of iron deficiency on the digestive utilization of iron, phosphorus, calcium and magnesium in rats. Br. J. Nutr. 70:609-620.

Parkkila, S., O. Niemela, E. R. Savolainen, and P. Koistinen. 2001. HFE mutations do not account for transfusional iron overload in patients with acute myeloid leukemia. Transfusion 41:828-831.

Pawlotsky, Y., P. Le Dantec, R. Moirand, P. Guggenbuhl, A. M. Jouanolle, M. Catheline, J. Meadeb, P. Brissot, Y. Deugnier, and G. Chalès. 1999. Elevated parathyroid hormone 44-68 and osteoarticular changes in patients with genetic hemochromatosis. Arthritis Rheum. 42:799-806.

Raja, K. B., R. J. Simpson, and T. J. Peters. 1994. Intestinal iron absorption studies in mouse models of iron-overload. Br. J. Haematol. 86:156-162.

Reeves, P. G., F. H. Nielsen, and G. C. Fahey. 1993. AIN-93 Purified diets for laboratory rodents: Final report of the American Institute of Nutrition and Ad Hoc Writing Committee on the reformulation of the AIN-76A rodent diet. J. Nutr. 123:1939-1951.

Rissanen, J. P., M. I. Suominen, R. Oksala, L. Ravanti, P. Kallio, and J. M. Halleen. 2006. The ratio of osteoclast activity/osteoclast number (CTX/TRACP 5b) is a useful parameter in experimental rat models of osteoporosis. J. Bone Miner. Res. 21:SU105.

Shawki, A., and B. Mackenzie. 2010. Interaction of calcium with the human divalent metal-ion transporter-1. Biochem. Biophys. Res. Commun. 393:471-475.

Tuderman, L., R. Myllo, and K. I. Kivirikko. 1977. Mechanism of the prolyl hydroxylase reaction. I. Role of co-substrates. Eur. J. Biochem. 80:341-348. 